\title{
New Binary and Exoplanet Candidates from STEREO Light Curves
}

\author{
Gemma Whittaker, Vino Sangaralingam and Ian Stevens \\ Astrophysics and Space Research, The University of Birmingham, Edgbaston, \\ Birmingham, B15 2TT, UK \\ email: gemma@star.sr.bham.ac.uk
}

\begin{abstract}
The Heliospheric Imagers (HI) onboard the STEREO satellites are observing an abundance of background stars as they follow their respective Sun-centered orbits. These are wide-angled CCD cameras with a $20^{\circ} \times 20^{\circ}$ field of view, directed $\sim 14^{\circ}$ from the solar disk. These imagers monitor $20 \%$ of the sky over one year, providing light curves for over 500,000 stars down to 12 th magnitude and brighter than 7 th. We are currently analysing the photometric data from the HI-1 cameras, obtained since March 2007. Following a standard data reduction of the raw photometric images, the resultant light curves underwent a sequence of detrending procedures to minimize systematics in the data, which can contribute to red noise. A transit search was performed using the BLS algorithm, which is sensitive to the box-like shape associated with planetary transits. The resulting candidates were subjected to a number of false-alarm tests to determine the most promising candidates and these were investigated further, visually and using available catalogue data. Possible new exoplanet and binary candidates will now be submitted for follow-up photometric and spectroscopic observations to confirm their nature.
\end{abstract}

Keywords. techniques: image processing, techniques: photometric, binaries: eclipsing, planetary systems, catalogs, methods: data analysis

\section{STEREO Observations}

STEREO (Solar TErrestrial RElations Observatory) was launched in March 2006 to study Coronal Mass Ejections (CMEs) in unprecedented detail by providing a stereoscopic view of the Sun. The twin observatories follow a heliocentric orbit, with one moving ahead of the Earth (STEREO-A) and the second lagging behind the Earth (STEREO-B). Among the four, identical suites of instruments onboard the two spacecraft is the SECCHI package (Sun Earth Connection Coronal and Heliospheric Investigation). SECCHI is comprised of an extreme UV imager, two optical coronagraphs and two heliospheric imagers (HI-1 and HI-2), designed (primarily) to study the 3-D evolution of CMEs along the Sun-Earth line.

The HI- 1 instruments are optical CCD cameras with a $20^{\circ} \times 20^{\circ}$ field of view. Within this field an estimated 29,000 stars between 7 th and 12th magnitude may be visible in the background at any one time. These cameras have a 40 minute observing cadence, which provides 36 consecutive frames per day for 20 days after which a star will pass out of the field of view. STEREO has now been fully operational for over 4 years, hence most stars have been observed at least 4 times by each camera. This has provided a wealth of precision, photometric data for many stars which are not well studied at present. 


\section{Data Processing and Detrending}

A standard data reduction is carried out for the raw photometric data, which are downloaded directly from the STEREO webpaget. This includes corrections for shutterless readout and saturated columns. Background subtraction is then performed to minimise contamination from the F-corona, using a 1-day running, minimum background. The NOMAD catalogue is used to track individual stars over consecutive frames and finally the light curves for these stars are extracted using aperture photometry. The HI-1 cameras have a moderate PSF of 3.7 arcmin (Cf. CoRoTs PSF which is 10.5 arcmin), meaning that the extracted light curves are susceptible to contamination from nearby stars.

\section{Transit Analysis and Results}

A transit search was performed, within a period range of 0.5 - 10 days, using the BLS transit detection algorithm (Kovács et al., 2002). From an initial study including 82,863 light curves from HI-1A, 952 potential candidates were detected with a Signal Detection Efficiency (SDE) of greater than 9. A series of false-alarm tests were then used to determine the most promising candidates. These included spectral-type information from SIMBAD and the Exoplanet Diagnostic Tool, $\eta^{*}$, (Tingley \& Sackett, 2005), which calculates the likelihood of a transit being from a planet as opposed to it being from a star or as a result of noise. The candidates passing all false-alarm tests were visually analysed to enable them to be classified into potential exoplanet or binary candidates.

Some binary targets have recently been submitted for preliminary spectroscopic followup using the WIRO telescope in Wyoming, USA. Present and future work is now focussed establishing a reliable set of targets for high resolution follow-up observations. This work will involve refining our search parameters and detrending procedures and the addition of the HI-1B data, which will increase the detection efficiency of BLS.

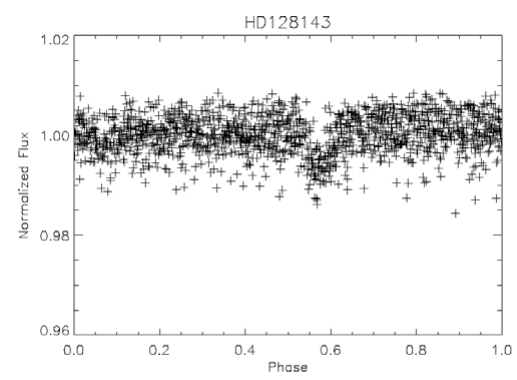

(a)

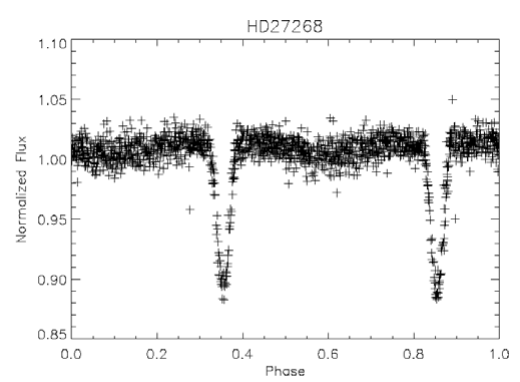

(b)

Figure 1. Example exoplanet and binary candidates. Figure (a) shows an F5 dwarf, which was detected by BLS with $S D E=15$, Period $=0.73$ days and $\eta^{*}=0.12$, which lies well below the cutoff value of 1.0 (indicative of a planet). Figure (b) shows an A0 star, which was detected with $S D E=40$, Period $=2.1$ days and $\eta^{*}=0.96$.

\section{References}

Kovács, G., Zucker, S. \& Mazek, T. 2002 A $\& A, 391,369$

Tingley, B. \& Sackett, P. 2005 ApJ, 627, 1011

Sangaralingam, V. \& Stevens, I. 2011 submitted

Whittaker, G., Sangaralingam, V. \& Stevens, I. 2011 in preparation

$\dagger$ www.ukssdc.rl.ac.uk/solar/stereo/data.html 